\title{
Сравнительный анализ методов оценки характеристик молекулярно-массового распределения полиметиленнафталинсульфонатов
}

\author{
Вовк А.И., Замуруев О.В. \\ Научно-технический цеентр ООО «Полипласт Новомосковск», Новомосковск
}

Поступила в редакцию 21.06.2016 г.

\begin{abstract}
Для технических полиметиленнафталинсульфонатов, применяемых в строительной промышленности, проведено сопоставление двух хроматографических методов оценки молекулярномассового распределения (ММР): высокоэффективной жидкостной (ВЭЖХ) и гель-проникающей хроматографии (ГПХ). Установлена предпочтительность метода ион-парной ВЭЖХ для получения надежных характеристик.
\end{abstract}

Ключевые слова: молекулярно-массовое распределение, хроматография, полиметиленнафталинсульфонаты, фракционирование

\section{Benchmarking study of methods for evaluating the molecular-weight distribution of polymethylene naphthalene sulfonates}

\author{
Vovk A.I., Zamuruev O.V. \\ $R \& D$ Centre Polyplast Novomoskovsk Ltd, Novomoskovsk
}

\begin{abstract}
Paper reviews briefly state of the art of research for polymethylene naphthalene sulfonate (PNS) properties. It was demonstrated that rates of PNS's molecular weight in technical publications may differ in order of magnitude or more. Two methods of molecular-weight distribution (HPLC, GPC) for technical PNS, used as construction chemicals, were compared. HPLC- and GPC-methods for estimation PNS molecularweight distribution were provided and the capabilities and main problems of both methods were highlighted. Insufficient PNS separation in GPC was demonstrated, that leads for incorrect molecular weight assessment. Ion-pairing HPLC provides the maximal separation selectivity of PNS homologous compounds (as compared to other methods) with analysis time less than 80 min. Ion-pairing HPLC provides an opportunity to qualify sodium naphthalene sulfonates, disodium naphthalene disulfonates and a lot of PNS. On the base of empirical data, extensive analysis of PNS chromatographic behavior has been carried out. Molecular-weight characteristics for some PNS-based superplasticizers were calculated: $M_{\mathrm{n}} 2779-2920 \mathrm{Da}, M_{\mathrm{w}} 3291-3355 \mathrm{Da}$, polydispersity index 1.15-1.21. The data obtained may be used to predict various technical PNS performance in cement systems (cement paste, mortar, fresh concrete). The developed HPLC methods may considered as suitable quick-look method of production control.
\end{abstract} fractionation

Keywords: molecular-weight distribution, chromatography, polymethylene naphthalene sulfonates,

\section{Введение}

Известна зависимость пластифицирующего действия полиметиленнафталинсульфонатов (ПНС) в цементном тесте, растворной и бетонной смесях от их молеку- 
лярной массы [1]. Представлены публикации с аналогичными выводами и для других полимеров с неизменной структурой элементарного звена: сульфированных меламинформальдегидных смол и полистиролсульфонатов [2]. Что касается нового, весьма популярного сейчас класса суперпластификаторов - полиэфиркарбоксилатов (ПЭК), то они, во-первых, представляют полимеры со статистическим распределением мономерных звеньев (т.е. не содержат элементарных звеньев постоянной длины и неизменной формулы) и, во-вторых, их свойства в значительной степени зависят от длины привитых боковых цепей. Поэтому свойства ПЭК не могут однозначно соотноситься с молекулярной массой полимера и зависят от многих факторов, в том числе, от соотношения компонентов, условий синтеза (равномерности прививки) и молекулярной массы макромономера.

Для ПНС, как и для всех полимеров могут применяться любые классические методы [3] прямого определения средних значений молекулярной массы: среднечисловой $\left(M_{\mathrm{n}}\right)$ - через коллигативные характеристики; среднемассовой $\left(M_{\mathrm{w}}\right)$ - по светорассеиванию; средневязкостной $\left(M_{\mathrm{v}}\right)$ - по вязкости. Однако намного более информативной является полноценная кривая молекулярно-массового распределения (MMP).

Анализ публикаций за последнее десятилетие показывает, что наиболее часто для характеристики МMP суперпластификаторов используют метод гельпроникающей хроматографии (ГПХ). Типичный вид гель-хроматограммы ПНС приведен в [4]. По мнению авторов, метод ион-парной высокоэффективной хроматографии (ВЭЖХ) не менее перспективен для получения характеристик ММР соединений данного класса.

Целью представленной работы является сравнение возможностей ГПХ и ионпарной ВЭЖХ для оценки молекулярно-массового распределения полиметиленнафталинсульфонатов.

\section{Эксперимент}

Аппаратура. Хроматографический комплекс Knauer (Германия) серии Smartline состоял из: УФ-детектора (UV-Detector 2550), термостата колонок (Column Oven 4050), насоса (Pump 1050), управляющего блока с модулем дегазатора и модулем формирования градиента на стороне низкого давления (Manager 5050).

Метод ион-парной ВЭЖХ. Разделение проводили на хроматографической колонке $(250 \times 4.6$ мм), заполненной сорбентом Kromasil $100 \mathrm{C} 18$ (Dr. Maisch GmbH, Германия) с размером частиц 5 мкм. Температура термостата колонок $30{ }^{\circ} \mathrm{C}$. Объем хроматографируемой пробы, вводимой через кран-дозатор 20 мкл.

Использовали градиентное элюирование двухкомпонентной системой. Подвижные фазы формировали с использованием растворителей: A - ацетонитрил LiCh$\operatorname{rosolv}{ }^{\circledR}$, чистоты для градиентной хроматографии (EDM Millipore Corporation - division of Merck KGaA, Германия), В - дистиллированная вода (удельное сопротивление не менее 18 МОм·см). В растворители А и В добавлено 6 ммоль/л тетрабутиламмоний бромида (ч.д.а., Acros, США). Перед использованием растворители фильтровали на установке Sartorius Stedim Biotech (Германия) через мембранный фильтр с диметром пор 0.45 мкм. Градиент: 0 мин - 30 об.\% (А), 0-40 мин - 55 об.\% (А), 40-90 мин - 75 об.\% (А). Расход элюента $-1 \mathrm{~cm}^{3} /$ мин. Аналитическая длина волны детектора 290 нм.

В овк и др. / Сорбционные и хроматографические процессы. 2017. Т. 17. № 1 
Для приготовления аналитической пробы, навеску образца 0.5000 г переводили растворителем (A:B=30:70 об.\%) в мерную колбу емкостью $100 \mathrm{~cm}^{3}$, растворяли, разбавляли до метки и перемешивали.

Метод ГПХ. Разделение проводили в изократическом режиме элюирования на колонке Ultrahydrohel 250 (США, Waters Corp.) размером $300 \times 7.8$ мм, область разделения 1000-80000 Да. Объем хроматографируемой пробы, вводимой через крандозатор 20 мкл. В качестве элюента использовали 0.1 М раствор нитрата натрия, предварительно отфильтрованный через мембранный фильтр с диаметром пор 0.45 мкм. Расход подвижной фазы $0.8 \mathrm{~cm}^{3} /$ мин. Аналитическая длина волны детектора 290 HM.

Для приготовления хроматографической пробы навеску образца 0.5000-0.8000 г взвешивали в мерной колбе емкостью $50 \mathrm{~cm}^{3}$, доводили объем элюентом до метки и перемешивали до полного растворения.

\section{Обсуждение результатов}

Первые сведения о степени поликонденсации ПНС получены К. Хаттори [5]. Перевод ПНС в сульфохлориды с последующей бумажной хроматографией позволил выделить и идентифицировать соединения со степенью поликонденсации до $n=9$. Средняя молекулярная масса каждой выделенной фракции подтверждалась криоскопическим методом. При этом сведений о выделении изомеров не приводилось.

При отсутствии иных исследований длительное время данные результаты трактовали (и в отечественной, и в зарубежной литературе) как свидетельство олигомерного характера ПНС со степенью поликонденсации не более 9 [6]. Отсюда ряд авторов [7], следуя общепринятой точке зрения, произвольно соотносили интенсивный неразделенный пик на гель-хроматограмме с суммарным содержанием гепта-, окта- и нонамеров ПНС.

В конце 80-х за рубежом был опубликован ряд статей итальянских ученых $[8,9]$, а также работа [10], в которых были приведены оценки молекулярных масс ПНС. К этому моменту в нашей стране были предложены две методики изучения молекулярного состава ПНС и оценки их ММР, основанные на препаративной колоночной хроматографии и селективном экстрагировании с последующим осаждением целевой фракции [11]. Было показано, что в состав технических продуктов входят ПНС со степенями поликонденсации до $n \approx 20$, исходя из чего для серии технических продуктов были получены значения $M_{\mathrm{n}}$ 1300-1800 Да и $M_{\mathrm{w}}$ 2300-2600 Да. Селективность выделения индивидуальных полимергомологов ПНС при колоночной хроматографии и разделения фракций при экстракции-осаждении контролировалась методом ТСХ.

Полученные значения средних молекулярных масс ПНС и предполагаемое закономерное различие гидродинамических радиусов молекул олигомеров позволило предположить, что вещество должно эффективно разделяться в условиях ГПХ на Sefadex G25 (область разделения по полисахаридам 100-5000 Да). Полученный вид хроматограммы [12] качественно соответствовал результатам, представленным в работе [7]: наблюдался интенсивный неразделенный пик с последующим выходом 5-6 умеренно разделенных пиков, размытие которых, частично, можно отнести к возможному содержанию изомерных фракций. Различие состояло лишь в отнесении основного пика, который по нашим данным [12], основанным на изучении взаимосвязи строения ПНС и их ультрафиолетовых спектров, соответствовал олигомерам с $n \geq 10$.

Вовк и др. / Сорбционные и хроматографические процессы. 2017. Т. 17. № 1 
Дальнейшие исследования, направленные на повышение селективности разделения ПНС за счет перехода к сорбентам с большим размером пор, показали, что применение Sefadex G50 существенно не улучшило качество разделения, а использование Sefadex G100 (предел эксклюзии 100-150 кДа) даже его снизило. Это позволило сделать выводы о нецелесообразности применения сорбентов с еще большим размером пор. В работе [13] исследователями также показана малая эффективность применения метода ГПХ для исследования полимерных сульфокислот.

По-видимому, главной проблемой наблюдаемых несоответствий (размера пор геля и диапазона молекулярных масс ПНС) и причиной низкой селективности метода являются высокие значения гидродинамических радиусов фракций ПНС разной молекулярной массы.

Если не учитывать влияние фактора гидродинамического радиуса, то использование набора мембран с различным размером пор (соответствующих по данным разработчика молекулярным массам от 500 Да до 100 кДа) приводит к выводу о наличии в составе ПНС фракций с молекулярной массой более 100 кДа (до 35\%) [4]. При этом для двух образцов технического ПНС были вычислены следующие значения средних молекулярных масс: «низкоконденсированный» продукт - $M_{\mathrm{n}} 2900$ Да и $M_{\mathrm{w}} 32000$ Да; «высококонденсированный»- $M_{\mathrm{n}} 5800$ Да и $M_{\mathrm{w}} 75000$ Да. Если по приведенным данным рассчитать полидисперсность (ПД) 11.1 и 12.9, то видно, что получаются абсолютно не типичные значения для данного класса соединений (обычно величина ПД не превышает 2) [14]. Еще более высокие значения молекулярной массы ПНС, полученной по методу ГПХ, приводит Планк [15]: $M_{\mathrm{w}} \sim 139$ кДа. При этом для полиалкансульфонатной добавки (низкомолекулярного аналога ПНС) определенные им значения гидродинамического радиуса $\left(R_{\mathrm{h}}\right)$ и молекулярной массы $\left(M_{\mathrm{w}}\right)$, соответственно, составили: 7.8 нм и 27330 Да. В то же время, для заметно более высокомолекулярного ПЭК $\left(M_{\mathrm{w}}=60240\right.$ Да) значение $R_{\mathrm{h}}$ составило всего 6.7 нм.

Эти данные отчетливо подтверждают значительное увеличение гидродинамического радиуса для сульфонатных полимеров, соответственно, для них становятся непригодными калибровки, основанные на стандартных образцах полиэтиленгликоля (ПЭГ), которые наиболее часто применяют исследователи. Аналогичный вывод содержится и в статье [16].

С середины 70-х годов начало интенсивно развиваться новое направление в жидкостной хроматографии - ион-парная ВЭЖХ. Этот метод занимает промежуточное положение между классическими ионообменной и обращенно-фазовой хроматографией и во многих случаях позволяет обеспечить более эффективное разделение компонентов.

Поскольку для ПНС количество сульфогрупп в молекуле совпадает со степенью поликонденсации (исследователями показано [17], что дисульфопроизводные практически не вступают в реакцию олигомеризации) то, обеспечив селективное разделение компонентов, можно количественно определить содержание каждого полимергомолога (с учетом суммы изомеров) и повысить точность расчета характеристик ММР.

В ходе исследований нами установлено, что максимальная селективность разделения ПНС достигается в градиентном элюировании [18] (рис. 1). При этом на хроматограмме можно выделить до 14-15 групп пиков, отвечающих выходу индивидуальных полимергомологов ПНС. Согласно теоретическим основам ВЭЖХ [19] времена удерживания компонентов смеси в условиях ион-парной хроматографии будут определяться комплексом достаточно сложных равновесных процессов, конкурирующих между собой. С одной стороны, за счет гидрофобных взаимодействий и эффекта вытеснения из полярной среды подвижной фазы возможна сорбция гидро- 
фобных ионов модификатора (тетрабутиламмония) на поверхности алкилсиликагеля таким образом, что алкильный фрагмент динамического модификатора прижат к поверхности, а заряженная группа обращена к подвижной фазе. В этом случае поверхность приобретает ионообменные свойства, и удерживание подчиняется закономерностям ионообменной хроматографии. С другой стороны, возможно образование ионной пары непосредственно в объеме элюента, которая затем сорбируется на неполярной поверхности по обращенно-фазовому механизму.

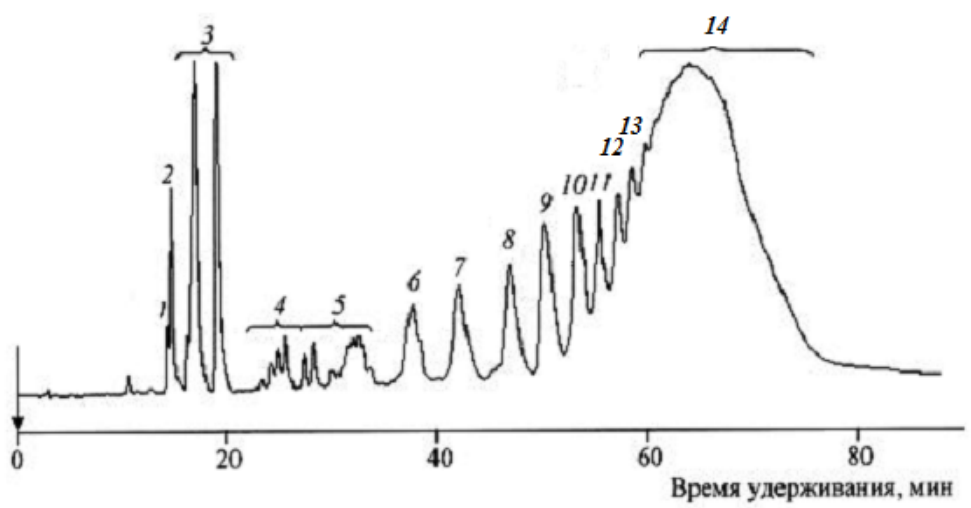

Рис. 1. Хроматограмма технического ПНС (табл. 1, образец 1), полученная методом ион-парной ВЭЖХ: 1 - Na-1-НСК; 2 - Na-2-НСК; 3 - НДСК; 4-14 - олигомерные фракции ПНС, соответственно, 4-13 при $n=2-11,14$ при $n \geq 12$

С учетом идентичности (поскольку предполагается, что дисульфопроизводные присутствуют преимущественно только в виде мономерных фракций [17]) химической структуры олигомерных компонентов разделяемой пробы (элементарное звено - метиленнафталинсульфонат) главными факторами, определяющими силу удерживания, являются: количество сульфогрупп, сила кислоты (при равенстве количества $\mathrm{SO}_{3} \mathrm{Na}-г р у п п$ - изомеры по $\alpha$ - и $\beta$-положению) и пространственная доступность (степень экранирования кислотных групп ароматическими кольцами), определяемая пространственной конформацией молекул ПНС.

В соответствии с этим предполагалось, что первыми должны элюироваться соединения, содержащие одно нафталиновое кольцо. Действительно, использование хроматографических свидетелей Na-соли 2-нафталинсульфокислоты (квалификация ч.), Na-соли 1-нафталинсульфокислоты и смеси ди-Na-солей 2,6- и 2,7нафталиндисульфокислоты (НДСК, синтезированы согласно [20]) позволило надежно идентифицировать 4 первых пика. Всем этим компонентам на хроматограмме соответствуют узкие симметричные пики (рис. 1).

Следующие за ними группы пиков (в диапазоне времен удерживания 22-40 мин) обладают сложной структурой. Мы полагаем, что это обусловлено структурной неоднородностью олигомерных ПНС (наличием изомеров положения как по сульфогруппе, так и по метиленовому мостику) [1]. Правильность отнесения пиков к полимергомологам ПНС была качественно подтверждена хроматографированием фракций низко-, средне- и высокомолекулярных ПНС, выделенных по методике [11]. Результаты хроматографического разделения выделенных фракций представлены в табл. 1. Несмотря на то, что практически во всех фракциях присутствует полный набор компонентов пробы, отчетливо видно, что доля целевых компонентов в выделенных фракциях заметно возрастает.

На основании данных хроматографического разделения (табл. 2) рассчитали значения $M_{\mathrm{n}}, M_{\mathrm{w}}$ и ПД, который характеризует ширину ММР: 


$$
M_{n}=\frac{\Sigma\left(N_{i} M_{i}\right)}{\Sigma N_{i}} ; M_{W}=\frac{\Sigma\left(N_{i} M_{i}^{2}\right)}{\Sigma N_{i} M_{i}} ; \Pi \text { П }=\frac{M_{W}}{M_{n}},
$$

где $i=$ от 2 до 25 (верхний предел, на данный момент, принимается как общепринятая величина [21]).

Таблица 1. Результаты ВЭЖХ-анализа фракций, выделенных из технического образца ПНС (табл. 2, образец 1) в соответствии с методикой [11]

\begin{tabular}{|c|c|c|c|c|c|c|}
\hline \multirow{2}{*}{$\begin{array}{c}\text { Диапазон вре- } \\
\text { мен удержива- } \\
\text { ния целевых } \\
\text { компонентов } \\
\text { фракции, мин }\end{array}$} & \multicolumn{6}{|c|}{$\begin{array}{l}\text { Определяемая группа веществ в хроматографической пробе и ее содер- } \\
\text { жание*, \% }\end{array}$} \\
\hline & HCK & НДСК & $n=2-4$ & $n=5-9$ & $n=10-11$ & $\geq 12$ \\
\hline \multicolumn{7}{|c|}{ «низкомолекулярная» фракция } \\
\hline $22-38$ & 22.3 & 28.3 & 21.8 & 7.4 & 4.9 & 15.3 \\
\hline \multicolumn{7}{|c|}{ «среднемолекулярная» фракция (целевые компоненты: олигомеры с $n=5-9$ ) } \\
\hline $42-36$ & 7.9 & 13.5 & 10.1 & 28.8 & 6.1 & 33.6 \\
\hline \multicolumn{7}{|c|}{ «высокомолекулярная 1» фракция (целевые компоненты: олигомеры с $n=10-11$ ) } \\
\hline $57-59$ & 2.2 & 4.8 & 10.3 & 12.9 & 24.1 & 45.7 \\
\hline \multicolumn{7}{|c|}{ олекулярная 2» фракция (целевые компоненты: олигомеры с $n \geq 12$ ) } \\
\hline$\geq 60$ & 0.5 & 1.5 & 2.7 & 5.5 & 5.6 & 84.2 \\
\hline
\end{tabular}

* Сумма всех компонентов на хроматограмме приведена к $100 \%$.

Условное отнесение неразрешенного пика полимерных ПНС (с максимумом элюирования более 60 мин) к соединениям с $12 \leq n \leq 25$ приводит для ряда технических ПНС к значениям $M_{\mathrm{n}}$ 2779-2920 Да, $M_{\mathrm{w}}$ 3291-3355 Да и ПД 1.15-1.21 (табл. 2).

Таблица 2. Результаты ВЭЖХ-анализа промышленных образцов технических ПНС

\begin{tabular}{|c|c|c|c|c|c|c|c|}
\hline \multirow{2}{*}{$\begin{array}{c}\text { Определяемая группа ве- } \\
\text { ществ в хроматографиче- } \\
\text { ской пробе и ее содержа- } \\
\text { ние*, \% }\end{array}$} & \multicolumn{7}{|c|}{ Номер ПНС } \\
\hline & 1 & 2 & 3 & 4 & 5 & 6 & 7 \\
\hline $\begin{array}{l}\text { - сумма НСК и НДСК } \\
\text { - олигомерные фракции: }\end{array}$ & 8.1 & 7.2 & 6.4 & 6.7 & 7.6 & 7.4 & 9.5 \\
\hline$n=2-4$ & 12.3 & 6.8 & 8.3 & 9.8 & 6.3 & 6.5 & 7.7 \\
\hline$n=5-8$ & 19.6 & 9.8 & 10.7 & 11.5 & 8.8 & 9.6 & 10.2 \\
\hline$n \geq 9$ & 60.0 & 76.2 & 74.6 & 72.0 & 77.3 & 76.5 & 72.6 \\
\hline \multicolumn{8}{|c|}{ Параметры молекулярно-массового распределения ПНС } \\
\hline$M_{\mathrm{n}}$, Да & 2946 & 2899 & 2865 & 2797 & 2920 & 2905 & 2779 \\
\hline$M_{\mathrm{w}}$, Да & 3498 & 3335 & 3316 & 3291 & 3348 & 3338 & 3355 \\
\hline ПД & 1.19 & 1.15 & 1.16 & 1.18 & 1.15 & 1.15 & 1.21 \\
\hline
\end{tabular}

* Сумма всех компонентов на хроматограмме приведена к 100\%.

Переход от ион-парной ВЭЖХ к высокоэффективной эксклюзионной хроматографии значительно ухудшил селективность разделения ПНС. Достаточно низкую селективность разделения хроматографической пробы в условиях ГПХ можно было бы объяснить наличием агрегации молекул ПНС по мицеллярному типу. Однако, измерение критической концентрации мицеллообразования (ККМ) показало, что концентрация вещества в хроматографической пробе (10-16 г/дм³), значительно меньших ККМ (211.1 г/дм³ ных фракций ПНС с преимущественным содержанием олигомеров $n \geq 12$ ). 
На хроматограмме того же самого (табл. 2, образец 1) технического ПНС наблюдается всего 2 пика с временами выхода 9.3 и 13.3 мин и соотношением площадей $~ 30: 70$ (рис. $2 a$ ). Первый пик - уширенный и асимметричный, предположительно соответствующий высокомолекулярным олигомерам; второй - узкий и симметричный, предположительно соответствующий мономерам и низкомолекулярным олигомерам. Для уточнения отнесения пиков были проанализированы образцы узких фракций ПНС, полученные по методике [11]. При хроматографировании «низкомолекулярной» фракции (молекулярная масса тетрамера формально соответствует нижнему заявленному пределу разделения колонки) наблюдается только пик с временем выхода 13.3 мин (рис. 2б). Хроматографирование выделенной «высокомолекулярной 2» фракций ПНС (рис. 26) дало те же два аналитических отклика, но с соотношением площадей $~ 60: 40$. Вызывает некоторое недоумение столь высокий аналитический сигнал, соответствующий мономерным и низкомолекулярным олигомерным продуктам, особенно, с учетом результатов анализа этой же пробы методом ион-парной ВЭЖХ.
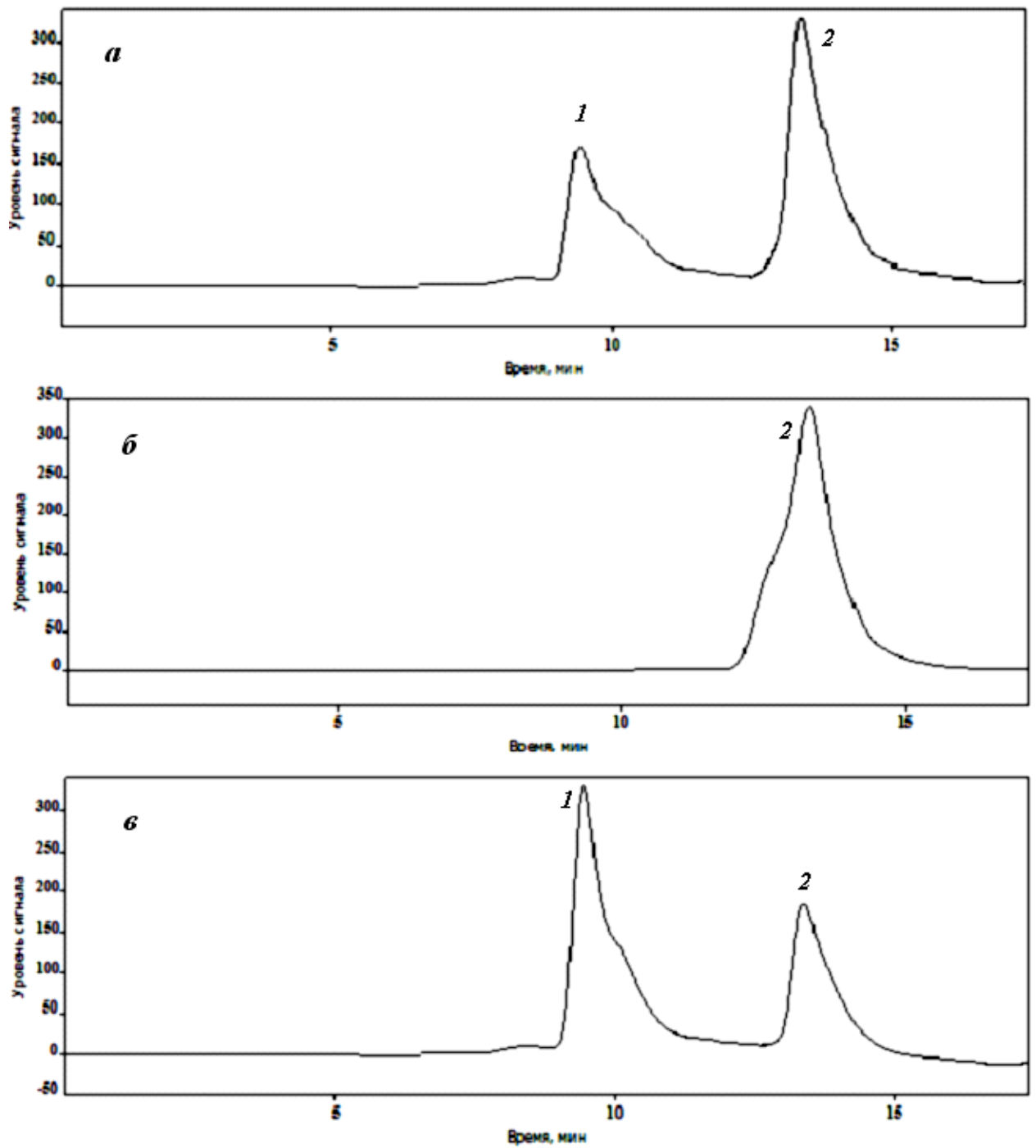

Рис. 2. Хроматограммы, полученные методом ГПХ: $a$ - технического ПНС; $\sigma$ - «низкомолекулярной» фракции выделенной из технического ПНС; 8 - «высокомолекулярной 2» фракции выделенной фракции из технического ПНС.

1 - высоко- и 2 - низкомолекулярные компоненты технического ПНС 
При калибровке колонки по полиэтиленгликолям, молекулярная масса «высокомолекулярной 2» фракции ПНС составила 8-10 кДа, что соответствует диапазону степеней поликонденсации $n=33-40$.

\section{Заключение}

Показано, что ион-парная ВЭЖХ обеспечивает большую селективность разделения полимергомологов ПНС по сравнению с ГПХ при удовлетворительной продолжительности анализа. Вычислены показатели МMP для ряда суперпластификаторов на основе ПНС.

\section{Список литературы}

1. Вовк А.И. Дисс. канд. техн. наук. М. 1987. $252 \mathrm{c}$.

2. Мамедов М.М. Дисс. канд. хим. наук. Баку. 1989. 22 с.

3. Фракционирование полимеров. / Под ред. Кантова М. Пер. с англ. под ред. Н.С. Наметкина, А.Д. Литмановича. М. Мир. $1971.444 \mathrm{c}$.

4. Spiratos N., Pagé M. Mailvaganam N. et al. Superplasticizers for Concrete: Fundamentals, Technology and Practice. Quebec. Canada. 2006. $322 \mathrm{p}$.

5. Hattory K. // Kagaku gijutsu. 1976. Vol. 29. No 8, pp. 10-21.

6. Иванов Ф.М., Москвин В.М, Батраков В.Г. и др. // Бетон и железобетон. 1978. №10. C. 13-16.

7. Garvey M.J., Tadros T.F. // KolloidZeitschrift und Zeitschrift fur polymere. 1972. Bd. 250. No 10. pp. 967-972.

8. Basile F., Biangini F., Ferrari G., Collepardi M. // 3-rd CANMET/ACI International conference. Ottawa, Canada. 1989. pp. 209-220.

9. Ferrari G., Basile F., Dal'Bo A., Mantoni A. // Cemento. 1986. Vol. 83. No 4. pp. 445-454.

10. Pieh S. // Angew. Makromol. Chem. 1987. Bd. 154. pp.145-159.

11. Рекомендации по физико-химическому контролю состава и качества суперпластификатора С-3. М. НИИЖБ. 1984. 56 с.

12. Вовк А.И. Дисс. докт. техн. наук. М. 1994. $425 \mathrm{c}$.

\section{References}

1. Vovk A.I. Diss. kand. tekhn. nauk. M., 1987, $252 \mathrm{p}$.
13. Roy D.M., Varadi G., Tamas F. et al. // Cem. and Concr. Res. 1984. Vol. 19. No 3. pp. 439-442.

14. Краснова Т.А. Дисс. канд. хим. наук. Саратов. 2013. 147 c.

15. Lei L., de Reese J., Plank. J. // «Superplasticizers and Other Chemical Admixture in Concrete». Proceedings Tenth International Conference. .Prague. 2012. pp. 11-28.

16. Albrecht G., Vierle M. // «The 9th ACI International Conference on Superplasticizers and Other Chemical Admixtures». Proceedings Ninth ACI International Conference.Seville. 2009. pp. 321-334.

17. Basile F., Biagini S., Ferrari G., Collepardi M. // «Superplasticizers and Other Chemical Admixture in Concrete». Proceedings Third International Conference. 1989. Ottawa. 1989. pp. 209-221.

18. Замуруев О.В., Петрович О.М., Тюрина Л.А., Вовк А.И. // Журнал аналитической химии. 2015. Т. 70. №. 12. С. 1521-1525

19. Стыскин Е.Л., Ициксон Л.Б, Брауде Е.В. Практическая высокоэффективная жидкостная хроматография. М. Химия. 1986. 213 c.

20. Дональдсон Н. Химия и технология соединений нафталинового ряда. М. Госхимиздат. $1963.656 \mathrm{c}$.

21. ТУ 5870-005-58042865-05. Суперпластификатор «Полипласт СП-1». Технические условия. М. ОАО «Полипласт». 2005. 19 с.

2. Mamedov M.M. Diss. kand. khim. nauk. Baku, 1989, 22 p. 
3. Fraktsionirovanie polimerov / Pod red. Kantova M. Per. s angl. pod red. N.S. Nametkina, A.D. Litmanovicha, M., Mir, 1971, 444 p.

4. Spiratos N., Pagé M. Mailvaganam N. et al. Superplasticizers for Concrete: Fundamentals, Technology and Practice. Quebec, Canada, 2006, $322 \mathrm{p}$.

5. Hattory K. // Kagaku gijutsu., 1976, Vol. 29, No 8, pp. 10-21.

6. Ivanov F.M., Moskvin V.M, Batrakov V.G. et al. // Beton i zhelezobeton, 1978, No 10, pp. 13-16.

7. Garvey M.J., Tadros T.F. // KolloidZeitschrift und Zeitschrift fur polymere, 1972, Bd. 250, No 10, pp. 967-972.

8. Basile F., Biangini F., Ferrari G., Collepardi M. // 3-rd CANMET/ACI International conference. Ottawa, Canada, 1989, pp. 209-220.

9. Ferrari G., Basile F., Dal'Bo A., Mantoni A. // Cemento, 1986, Vol. 83, No 4, pp. 445-454.

10. Pieh S. // Angew. Makromol. Chem., 1987, Bd. 154, pp.145-159.

11. Rekomendatsii po fiziko-khimicheskomu kontrolyu sostava i kachestva superplastifikatora S-3. M/, NIIZhB, 1984, 56 p.

12. Vovk A.I. Diss. dokt. tekhn. nauk. M/, $1994,425 \mathrm{p}$.

13. Roy D.M., Varadi G., Tamas F. et al. // Cem. and Concr. Res., 1984, Vol. 19, No 3, pp.439-442.

Вовк Анатолий Иванович - д.т.н., директор Научно-технического центра ООО «Полипласт Новомосковск», Новомосковск

Замуруев Олег Викторович - к.Х.Н., зам.директора Научно-технического центра ООО «Полипласт Новомосковск», Новомосковск
14. Krasnova T.A. Diss. kand. khim. nauk. Saratov, 2013, $147 \mathrm{p}$.

15. Lei L., de Reese J., Plank. J. // "Superplasticizers and Other Chemical Admixture in Concrete", Proceedings Tenth International Conference, October 2012, Prague, 2012, pp. 11-28.

16. Albrecht G., Vierle M. // "The 9th ACI International Conference on Superplasticizers and Other Chemical Admixtures", Proceedings Ninth ACI International Conference, October 2009. Seville, 2009, pp. 321-334.

17. Basile F., Biagini S., Ferrari G., Collepardi M. // "Superplasticizers and Other Chemical Admixture in Concrete", Proceedings Third International Conference, October 1989, Ottawa, 1989, pp. 209-221.

18. Zamuruev O.V., Petrovich O.M., Tyurina L.A., Vovk A.I. // Journal of Analytical Chemistry, 2015, Vol. 70, No 12, pp. 1521-1525

19. Styskin E.L., Itsikson L.B, Braude E.V. Prakticheskaya vysokoeffektivnaya zhidkostnaya khromatografiya. Moskva, Khimiya, 1986, $213 \mathrm{p}$.

20. Donal'dson N. Khimiya i tekhnologiya soedinenii naftalinovogo ryada. Moskva, Goskhimizdat, 1963, $656 \mathrm{p}$.

21. TU 5870-005-58042865-05. Superplastifikator «Poliplast SP-1». Tekhnicheskie usloviya. M.: OAO «Poliplast», 2005. 19 p.

Vovk Anatoly I. - grand Ph.D (technical), the director of R\&D Centre Polyplast Novomoskovsk Ltd

Zamuruev Oleg V. - Ph.D (chemistry), the deputy directorof R\&D Centre Polyplast Novomoskovsk Ltd, e-mail: Ozdmuruev@mail.ru 